\title{
Improved Hydrodynamics for USP Apparatus 2
}

Arnold H. Beckett, Tinh I. Duach

Biovail Corporation International, Toronto, Canada

Glenn S. Kurs

VanKel Industries, Inc. Edison, N7

\section{Ahstract}

In spite of its growing importance in the modern dissolution laboratory, USP Apparatus 2 bas been plagued by many problems of reproducibility and accuracy which lead to wasted time using calibrators even when there is nothing wrong with the calibrators or the instruments. The primary source of variability bas been found to be in the bydrodynamics of the conventional cylindrical (i.e. USP bemispherical) vessel especially under the rotating paddle.

A new vessel, called the PEAK vessel, bas been developed which leads to better reproducibility and accelerated dissolution rates at comparable paddle speeds. The PEAK vessel eliminates the need for cumbersome deaeration, overcomes the problems caused by the differences in characteristics of currently manufactured band made vessels, and allows for the use of sampling probes of different sizes and characteristics in different locations in the vessels.

\section{Intraduction}

USP Apparatus 2 (paddle method) has become the workhorse instrument in the modern dissolution laboratory. It is expected to provide rugged and reproducible results, on which decisions about product quality, bioavailability, bioequivalence etc. are based. However, in practice, the apparatus tends to be extremely sensitive to outside and inside variables, with large changes in dissolution profiles resulting from small changes in factors such as paddle rotational speed, vibration, deaeration and insertion of sampling probes $(1,2)$. This can render results meaningless or impossible to interpret and thus consume enormous amounts of precious analyst time in investigating, correcting and revalidating the systems. It is largely for this reason that dissolution testing is usually regarded as nothing more than a mandated "service tool" of questionable performance used only to satisfy regulatory requirements, rather than a powerful predictive tool for pharmaceutical research and development (3).

\section{Hydrodynamic Problems}

Recent dissolution studies by Wang and Coffin (4) have shown that the commonly used rotation speed of $50 \mathrm{rpm}$ for tablet dissolution can create cones of insoluble materials on the bottom of the vessel.This cone is the result of the radial fluid flow present in USP cylindrical vessels. Invisible to the analyst's eye unless solids are

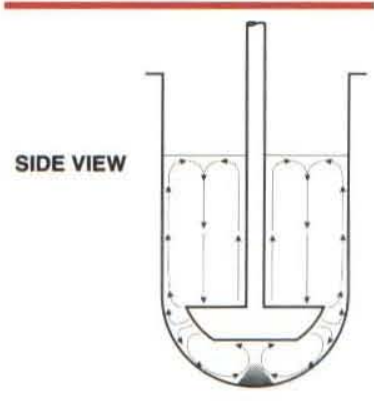

Figure 1. USP Vessel most of the material which accumulates inside is effectively shielded from the bulk of the present, this unstirred region is clearly visible when disintegrating tablets are used, as evidenced by a large and clearly defined cone of disintegrated material (Figure 1). Much of the variability in dissolution rates observed using Apparatus 2 is attributable to this cone, since moving medium in the vessel. Therefore, any external factor which alters the cone, such as speed changes, vibration, type and duration of deaeration or inserted sample probe, can have a large influence on the dissolution outcome. Also, the invisible liquid cone around non-disintegrating tablets is affected similarly. The rate controlling step of drug release from the product to the bulk dissolution medium often becomes the rate of release from the cone rather than the rate of release under 


\section{Improved Hydrodynamics....cont.}

sink conditions, even though there is enough volume of liquid in the dissolution vessel to theoretically provide the desired condition.

\section{The Solution}

A new vessel has been developed which eliminates the unstirred cone, and as a result, the variability concomitant with it. The new PEAK (5) vessel has a cone molded into the bottom using an appropriate tool to control its dimensions accurately (Figure 2). The glass cone effectively displaces the unstirred cone, forcing the material tested into the region of appropriate hydrodynamics, where all surfaces of the product are constantly and uniformly exposed to the moving medium. Experimental results show that this simple modification eliminates the above stated problems as shown in the following examples.

\section{Stirring Speed and Deaeration}

Changes in stirring speed and the air bubbles which tend to form in nondeaerated media change the flow characteristics of the media in conventional cylindrical vessels and accelerate dispersion of the cone, leading to erratic increases in dissolution rates compared to thoroughly deaerated media (1). Because the cone is already effectively well dispersed in PEAK vessels, dissolution rates are not influenced by the presence of air bubbles, i.e., dissolution rates are the same, whether or not the medium is deaerated. Figures 3, 4, \& 5 show the results of trial tests in PEAK and conventional vessels using both the USP salicylic acid and prednisone calibrators as well as the NCDA\#2 $10 \mathrm{mg}$ prednisone tablets.

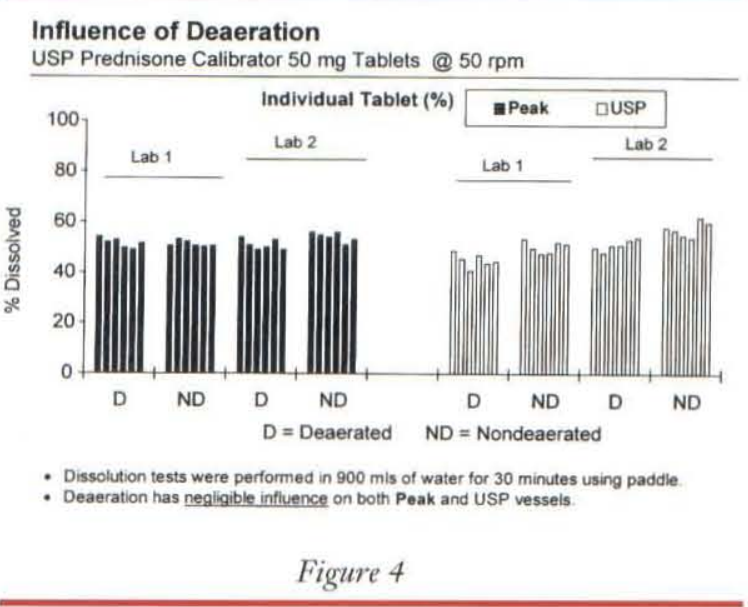

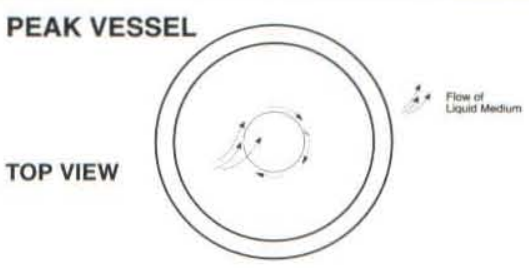

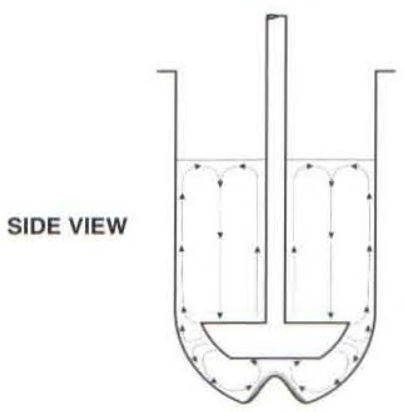

Figure 2. PEAK Vessel

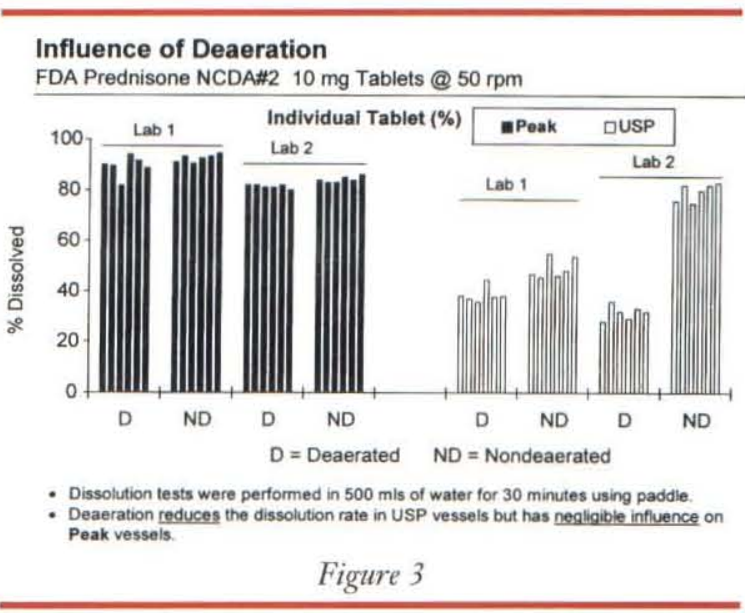

Influence of Deaeration

USP Salicyclic Acid Calibrator $300 \mathrm{mg}$ Tablets @ $50 \mathrm{rpm}$

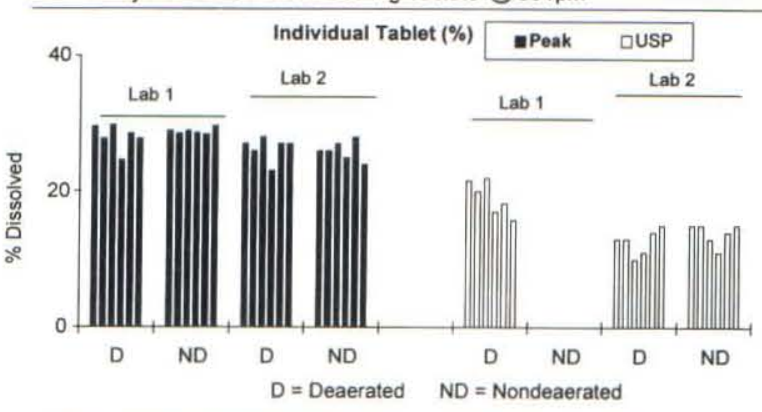

- Dissolution tests were performed in $900 \mathrm{mis}$ of $0.05 \mathrm{M}$ phosphate buffer $\mathrm{pH} 7.4$ for 30 minutes using paddle.

Figure 5 


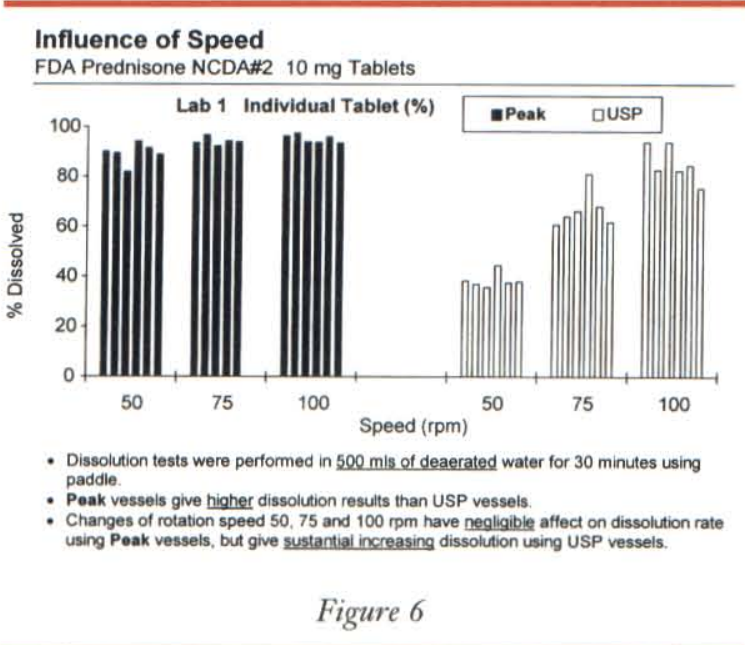

Conventional vessels exhibit marked differences in results between deaerated and nondeaerated media whereas PEAK vessels do not.

Using both PEAK and conventional vessels at speeds of 50, 75 and $100 \mathrm{rpm}$, the dissolution rates obtained in the former are at least twice that of the latter, are more reproducible, and differ little with changes in paddle rotational speed (Figures $6 \& 7$ ). In contrast, dissolution rates in conventional vessels were extremely sensitive to paddle

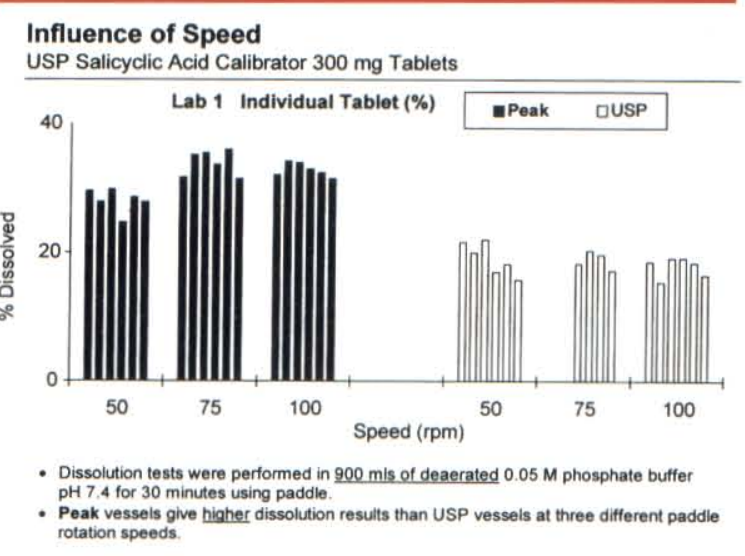

Figure 7

rotational speed. By inference, these data indicate that other factors such as internal or external vibration will have minimal effects on dissolution rates in PEAK vessels and to date no problems from vibration have been observed.

Data also indicate that dissolution is more complete in PEAK vessels compared to the conventional cylindrical type. It was found that after performing the dissolution of NCDA\#2 prednisone tablets for 60 minutes at $50 \mathrm{rpm}$, 


\section{Improved Hydrodynamics. ...ent.}

an increase in speed to $250 \mathrm{rpm}$ for an additional 60 minutes was required before all the drug was dissolved; this shows the great persistence of the cone after it has been established.

\section{Excellent Correlation to Dissolution Results Using USP Apparatus 3}

Figures 8, 9, \& 10 illustrate that dissolution results obtained from PEAK vessels in Apparatus 2 correlate closely with those from Apparatus 3. Thus the rate of release under truly sink conditions for either device is very similar, despite obvious outward differences in design, configuration, and movement pattern of the product in the two systems and their flow patterns.

\section{No Influence of Sampling Probes}

Until now, the use of sampling probes has tended to be problematic in USP Apparatus 2, since inserted probes perturb flow patterns and have a definite influence on dissolution rates. Wells has clearly demonstrated that the larger the sample probe, the larger the disturbance, and therefore, the higher the rate (2). This has forced the analysts to minimize both the size of sample probes and their residence time in the vessels. With the use of PEAK vessels, these concerns are eliminated. This will allow much greater freedom in sampling probe design, application and location of indwelling fiber optics, temperature, and $\mathrm{pH}$ probes in the vessels to facilitate recording information from each vessel during dissolution testing.

\section{Peak Vessels and usP Ealibrators}

The present USP $300 \mathrm{mg}$ salicylic acid nondisintegrating tablets are suitable for use in PEAK vessels; the corrected hydrodynamics will mean that the specifications currently used will need to be evaluated and adjusted upwards.

The present USP $50 \mathrm{mg}$ prednisone disintegrating tablets are unsuitable, as they are even for calibration of the conventional vessels. The NCDA $10 \mathrm{mg}$ prednisone tablet will be suit-

continued on page 18

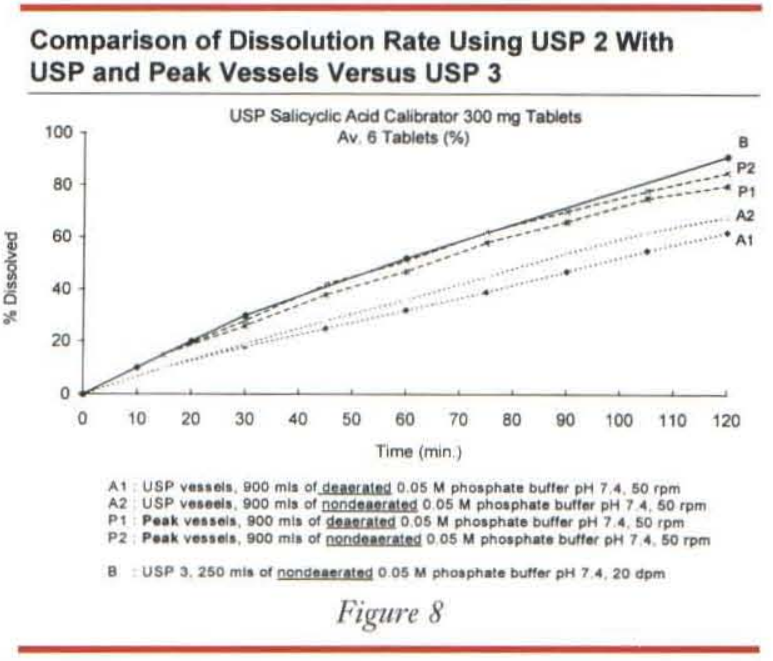

Comparison of Dissolution Rate Using USP 2 With USP and Peak Vessels Versus USP 3

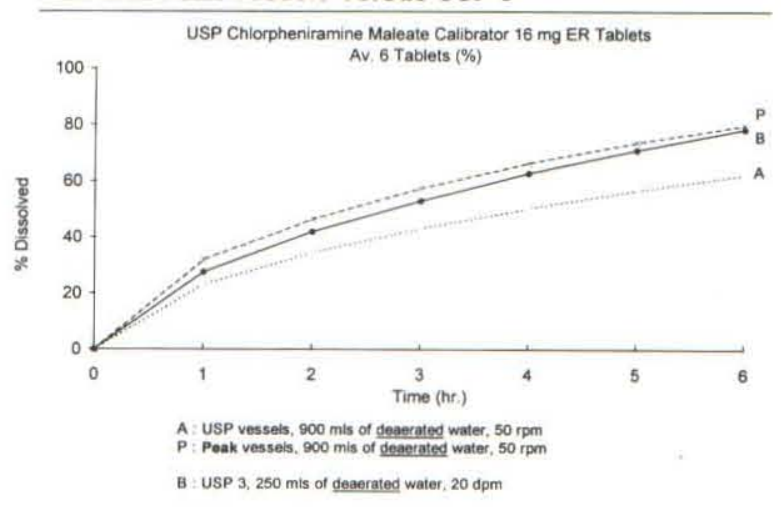

Figure 9

Comparison of Dissolution Rate Using USP 2 With USP and Peak Vessels Versus USP 3

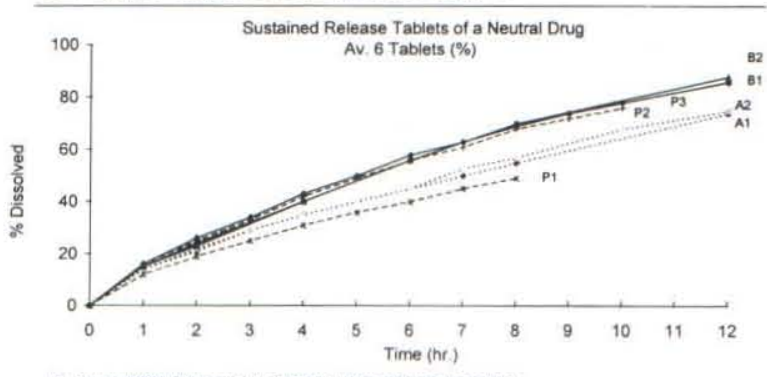

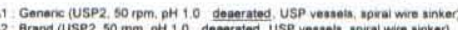

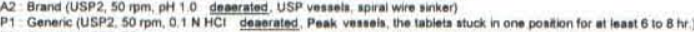

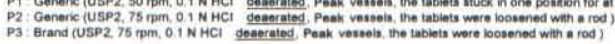

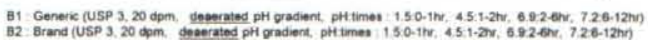

Figure 10 


\section{Improved Hydrodynamics....ent.}

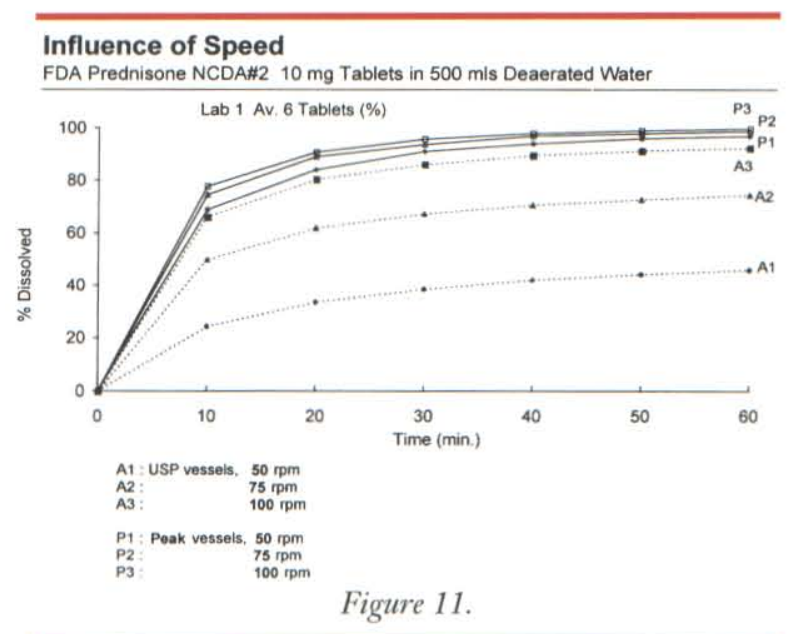

continued from page 10

able if its formulation is altered slightly to give a slower rate of drug release (Figure 11).

\section{Conclusions}

The PEAK vessel eliminates the hydrodynamic problems associated with conventional dissolution vessels by eliminating the unstirred region underneath the paddle. Disturbances to this region in conventional vessels result in significant variability in dissolution results; elimination of this region in PEAK vessels make dissolution rates insensitive to the usual external and internal sources of variation. The adoption of the PEAK vessel will result in higher degrees of confidence in results and minimization of the time and effort now expended in running USP system suitability tests.

Dissolution results will now represent more correctly the rate limiting step of drug release into solution under sink conditions. These will form a firm foundation for in vitro - in vivo correlation and the setting of meaningful drug release specifications for products based upon the use of the USP Apparatus 2.

\section{Acknowledgements}

The authors thank Dr. Ian Borst for analytical assistance and Mr. Mike Pocelinko for his support and assistance in the preparation of the manuscript.

\section{References}

(1) Moore T. W., Hamilton J. F., and Kerner C. M., Pharmacopeial Forum 1995; 21(5): 13871396

(2) Wells C. E., J. Pharm. Sci. 1982; 71(6): 232

(3) Beckett H. A., In-Vitro Dissolution Testing Technology, February 1996 Presentation at Vankel Industries Dissolution Communication and Exhibition, Edison, NJ, USA

(4) Wang T. and Coffin D. M., Glaxo Research Institute 1995 AAPS Poster Number APQ 1134

(5) PEAK Vessel ${ }^{\mathrm{TM}}$ \& patented by VanKel Industries, Inc. 